\title{
Unusual Burst Emission from the New Soft Gamma Repeater SGR1627-41
}

\author{
E.P.Mazets ${ }^{1,2}$, R.L.Aptekar ${ }^{1}$, P.S.Butterworth ${ }^{3,4}$, T.L.Cline ${ }^{3}$, D.D.Frederiks ${ }^{1}$, \\ S.V.Golenetskii ${ }^{1}$, K.Hurley ${ }^{5,6}$, V.N.Il'inskii ${ }^{1}$
}

\begin{abstract}
In June-July 1998 the Konus-Wind burst spectrometer observed a series of bursts from the new soft gamma repeater SGR 1627-41. Time histories and energy spectra of the bursts have been studied, revealing fluences and peak fluxes in the ranges of $3 \cdot 10^{-7}-7.5 \cdot 10^{-6} \mathrm{erg} \mathrm{cm}^{-2}$ and $10^{-5}-10^{-4} \mathrm{erg} \mathrm{cm}^{-2} \mathrm{~s}^{-1}$ respectively. One event, 18 June $6153.5 \mathrm{~s}$ UT stands out dramatically from this series. Its fluence is $\sim 7 \cdot 10^{-4} \mathrm{erg} \mathrm{cm}^{-2}$ and peak flux $\sim 2 \cdot 10^{-2} \mathrm{erg} \mathrm{cm}^{-2} \mathrm{~s}^{-1}$. These values from a source at a distance of $5.8 \mathrm{kpc}$ yield an energy output of $\sim 3 \cdot 10^{42} \mathrm{erg}$ and maximum luminosity of $\sim 8 \cdot 10^{43} \mathrm{erg} \mathrm{s}^{-1}$, similar to the values for the famous March 5, 1979 and August 27, 1998 events. In terms of energy, this event is another giant outburst seen in a third SGR! However, this very energetic burst differs significantly from the other giant outbursts. It exhibits no separate initial pulse with a fast rise time, no extended tail, and no pulsations. It is rather similar to ordinary repeated bursts but is a few hundred times stronger in intensity. According to the magnetar model by Thompson and Duncan (1995) such a burst may be initiated by a strong starquake when a crust fracture propagates over the whole surface of a neutron star.
\end{abstract}

\section{Introduction}

Repeating soft gamma-ray bursts were discovered 20 years ago (Golenetskii, Ilyinskii, \& Mazets, 1984; Mazets, Golenetskii, \& Guryan, 1979; Atteia et al. 1987). For a long time

\footnotetext{
${ }^{1}$ Ioffe Physico-Technical Institute, St.Petersburg, 194021, Russia.

${ }^{2}$ Mazets@pop.ioffe.rssi.ru

${ }^{3}$ Goddard Space Flight Center, Greenbelt, MD 20771, USA.

${ }^{4}$ Butterworth@lheavx.gsfc.nasa.gov

${ }^{5}$ Space Science Laboratory, University of California, Berkeley, CA 94720-7450, USA.

${ }^{6}$ KHurley@sunspot.ssl.berkeley.edu
} 
only three soft gamma repeaters were known (Norris, Hertz, \& Wood 1991) suggesting a rarity of this class of astrophysical objects (Kouveliotou et al. 1998). Two of them, SGR 1806-20 and SGR 1900+14, have exhibited reactivation phases after periods of long silence. Precise localizations and a search for optical counterparts revealed that all three SGRs are associated with rather young supernova remnants (Cline et al. 1982; Kulkarni et al. 1994; Hurley et al. 1999a) favoring a suggestion that the SGR's are neutron stars. Quiescent soft X-ray sources and emission periodicity were discovered associated with SGR 1806-20 (Murakami et al. 1994), SGR1900+14 (Hurley et al. 1999b) and SGR 0526-66 (Rothschild, Kulkarni \& Lingenfelter 1994). A spectacular huge periodic flare on August 27,1998 which came from SGR 1900+14 turned out to be strikingly similar to the famous event on March 5,1979. It demonstrated that such giant outbursts are an intrinsic but less common characteristic of SGRs (Cline, Mazets \& Golenetskii 1998). Observational data accumulated so far on SGRs have found their most complete explanation in the magnetar model by Thompson and Duncan (Thomson \& Duncan 1995) which proposes that SGRs are young slowly rotating neutron stars with superstrong magnetic fields of $\sim 10^{15} G$.

In June 1998, BATSE announced an observation of a fourth soft gamma repeater SGR 1627-41 (Kouveliotou et al. 1998 ) confirmed by Ulysses (Hurley, et al. 1998a), Beppo Sax (Feroci, et al. 1998), RXTE (Smith \& Levine 1998), and Konus-Wind (Hurley et al. 1998b) data. This SGR was precisely localized by IPN/Beppo Sax (Hurley et al. 1999d; Woods et al. 1999). Its position coincides with the SNR G337.0-0.1. Some evidence was obtained for a possible periodicity of $6.7 \mathrm{~s}$ (Dieters et al. 1998).

In this letter we report temporal and spectral properties of SGR 1627-41 as well as an unusual behavior of this source recorded in Konus-Wind observations.

\section{Observations}

The cosmic gamma-ray burst spectrometer Konus aboard the GGS WIND spacecraft observed 34 bursts from the new SGR in the period June 17-July 12,1998. Some events were too weak to trigger the instrument. They are recorded in a background mode with a time resolution of $2.94 \mathrm{~s}$ which is too coarse to study processes a small fraction of a second in duration. The bursts from the new source were strongly bunched in time. In two days, June,17 and 18, 29 bursts were observed. This high rate created an additional problem. The information on a burst which triggered the instrument is read by the S/C TM-system over a period of $\sim$ one hour. If another burst occurs in this time interval, it can't trigger the instrument and is recorded only among housekeeping data with $3.86 \mathrm{~s}$ resolution. As a

result, we obtained triggered records of only 13 bursts, which nevertheless give a good idea 
of the temporal and spectral behaviour of this SGR. Regrettably, as we can see from the untriggered data, several very interesting events were not caught with high time resolution.

In Figure 1 we show some examples of time histories of bursts recorded with a time resolution of $2 \mathrm{~ms}$ at photon energy $E_{\gamma}>15 \mathrm{keV}$. One can see that temporal structures are rather complex with rise and fall times of only a few milliseconds. Energy spectra, as expected, are soft with an exponential cutoff, $k T \sim 25 \mathrm{keV}$. Spectral variability is apparent in the course of many bursts. For example, time histories G1 and G2 of the burst on June 18, 16229.0s UT recorded in the low energy window G1(15 - $55 \mathrm{keV})$ and the middle one G2 $(55-250 \mathrm{keV})$ as well as the hardness ratio G2/G1 are shown in Figure 2. Detailed energy spectra for this event, accumulated after triggered time $T_{o}$ in subsequent $64 \mathrm{~ms}$ long time intervals, are presented in Figure 3. These photon spectra can be fitted by the expression $d N / d E \sim E^{-0.5} e^{-E / k T}$. A weak tail of the bursts exhibits a fading power law spectrum with an index of -2.8. Data obtained for 13 events are sampled in Table 1 which contains values of $\mathrm{kT}$, fluences, and peak fluxes; as well as the energy output and maximum luminosity of the source assuming a $5.8 \mathrm{kpc}$ distance (Case \& Bhattacharya 1998).

One event from this Table, GRB 980618a 6153.5s UT, stands out dramatically in intensity compared to the other bursts. The intensity of this event is so high that count rates in the low and middle energy windows approached saturation level. High count rates appear in the high energy window G3(250 - $1000 \mathrm{keV})$ due to the pile up of light pulses in the scintillator and photomultiplier. The instrument response to very high fluxes of various incident photon spectra were studied thoroughly in a laboratory simulation using a spare unit (Mazets, et al. 1999). This enables a reliable deconvolution of the Konus-Wind input fluxes. The time history of the giant outburst, after correction for dead time and pile up effects, is shown in Figure 4. Possible errors in the peak flux region can be represented by a scale factor of $0.5-1.5$.

Figure 5 presents energy loss spectra accumulated during four adjacent $64 \mathrm{~ms}$ long intervals and one of $256 \mathrm{~ms}$. The very hard spectrum in the 64-128 ms interval is partially the result of pile up of light flashes in the NaI crystal. From laboratory testing data however, it is evident that in this case the actual incident photon spectrum is also much harder than the spectrum for the first interval $T-T_{o}=0-64 \mathrm{~ms}$. Its $\mathrm{kT}$ can be as high as $100-150 \mathrm{keV}$ whereas unaffected spectra A, D, E correspond to $\mathrm{kT}: 50,50$, and $35 \mathrm{keV}$ respectively. 


\section{Discussion and conclusion}

The fluence and peak flux of this event exceed by several hundred times the values for other bursts of the series. At an assumed distance to the source of $5.8 \mathrm{kpc}$, they indicate an energy output of $\sim 3 \cdot 10^{42} \mathrm{erg}$ and a peak luminosity of $\sim 8 \cdot 10^{43} \mathrm{erg} \mathrm{s}^{-1}$. These quantities approach the huge energy releases and maximum luminosities for the giant outbursts on March 5,1979 and August 27,1998 (Hurley, et al. 1999c). From this point of view, the burst on June 18,1998 6153.5 s UT is also a giant outburst seen in a third SGR! But this burst differs strikingly from them. It exhibits no fast rise time, no long tail, and no pulsations. It is similar to ordinary repeating bursts from SGR 1627-41, but much stronger.

It is now widely accepted that an SGR's normal activity is a result of starquakes on neutron stars leading to a liberation of a large amount of the energy contained in superstrong magnetic fields of $\sim 10^{15} G$ (Thompson \& Duncan 1995). These authors have proposed that giant outbursts like the March 5 event are created by large scale reconnection instabilities of a huge stellar magnetic field. The more frequent weaker repeating bursts are generated during fractures of the neutron star crust. It was suggested that the energy of starquake-related bursts (i.e. their observed fluences) may depend on the size/length of fractures (Duncan, 1998; Golitsin, 1998). In such behavior an upper limit for the burst energy output will be determined by the maximum length of fractures crossing over the whole surface of the neutron star. It seems that the burst on June 18,1998 may be the first observed example of an event close to such an upper limit.

It is expected that rise times of repeated bursts must relate to their fluence $\mathrm{S}$ as $\tau \sim S^{1 / 3}$ (Golitsin, 1998). For weak bursts of (3-6) $10^{-7} \mathrm{erg} \mathrm{cm}^{-2}$ rise times are $\sim 10 \mathrm{~ms}$. For the giant event with $S \sim 2 \cdot 10^{-4} \mathrm{ergcm}^{-2}$ the rise time is $\sim 10^{2} \mathrm{~ms}$, in accord with the prediction.

There is some evidence that such energetic bursts may be quite common. Among bursts detected with Konus-Wind in untriggered background mode there are at least two events, June $1814360 \mathrm{~s}$ UT and June 1814661 s UT, with very high fluences. If their durations were short compared to the time resolution of $3.86 \mathrm{~s}$, then their intensities corrected for dead time could be comparable with the energy fluence of the strong June 18 burst under discussion.

These results provide an important confirmation of the SGR starquake model predictions.

This work on the Russian side was supported by RSA contract. 


\section{REFERENCES}

Atteia, J.-L., et al. 1987, ApJ, 320, L105

Case, G. L. \& Bhattacharya, D. 1998, ApJ, 504,761

Cline, T. L., et al. 1982, ApJ,255, L45

Cline, T. L., Mazets E. P., \& Golenetskii, S. V. 1998, IAU Circ. 7002

Dieters, S., et al. 1998, IAU Circ. 6962

Duncan,R.C. 1998, ApJ,498,L45

Golenetskii, S. V., Ilyinskii, V. N., \& Mazets, E. P. 1884, Nature, 307, 41

Golitsin, G. S. 1998, Astronomy Letters, 24(11),827

Feroci, M., et al. 1998, IAU Circ. 6945

Hurley, K., et al. 1998a, IAU Circ. 6948

Hurley, K., et al. 1998b, IAU Circ. 6966

Hurley, K., et al. 1999a, ApJ, 510, L107

Hurley, K., et al. 1999b, ApJ, 510, L111

Hurley, K., et al. 1999c, Nature, 397, 41

Hurley, K., et. al. 1999d, ApJ, in press

Kouveliotou, C., et al. 1994, Nature, 368, 125

Kouveliotou, C., et al. 1998, IAU Circ. 6944

Kulkarni, S. R., et al. 1994, Nature, 368, 129

Mazets, E. P., Golenetskii, S. V., \& Guryan, Yu. A. 1979, Soviet. Astron. Lett., 5(6), 343

Mazets, E.P. et al. 1999, Astronomy Letters, in press

Murakami, T., et al. 1994, Nature, 368,127

Norris, J. P., Hertz, P., \& Wood, K. S., 1991, ApJ, 366,240

Rothschild, R., Kulkarni, S., \& Lingenfelter, R. 1994, Nature, 368, 432

Smith, D. A., \& Levin, A.M. 1998, IAU Circ. 6950

Thompson, C., \& Duncan, R.C. 1995, MNRAS, 275,255

Woods, P., et al. 1999, Nature, in press 
Table 1. Main characteristics of observed bursts

\begin{tabular}{ccccccc}
\hline \hline GRB & $T_{o}(U T)$ & $\mathrm{kT}$ & $\mathrm{S}$ & $F_{\max }$ & $\mathrm{Q}$ & $\mathrm{L}$ \\
& $s$ & $\mathrm{keV}$ & $\mathrm{erg} \mathrm{cm} \mathrm{cm}^{-2}$ & $\mathrm{erg} \mathrm{cm}^{-2} \mathrm{~s}^{-1}$ & $\mathrm{erg} \mathrm{s}^{-1}$ \\
\hline $980617 \mathrm{a}$ & 71912.0 & 22 & $3.3 \cdot 10^{-6}$ & $3.7 \cdot 10^{-5}$ & $1.3 \cdot 10^{40}$ & $1.5 \cdot 10^{41}$ \\
$980617 \mathrm{~b}$ & 75880.0 & 14 & $1.2 \cdot 10^{-6}$ & $3.3 \cdot 10^{-5}$ & $4.8 \cdot 10^{39}$ & $1.3 \cdot 10^{41}$ \\
$980617 \mathrm{c}$ & 82449.0 & 18 & $7.0 \cdot 10^{-7}$ & $1.0 \cdot 10^{-5}$ & $2.8 \cdot 10^{39}$ & $4.0 \cdot 10^{40}$ \\
$980618 \mathrm{a}$ & 6153.5 & $\sim 150$ & $7.0 \cdot 10^{-4}$ & $2.0 \cdot 10^{-2}$ & $3.0 \cdot 10^{42}$ & $8.0 \cdot 10^{43}$ \\
$980618 \mathrm{~b}$ & 12316.5 & 16 & $6.5 \cdot 10^{-7}$ & $9.0 \cdot 10^{-6}$ & $2.6 \cdot 10^{39}$ & $3.6 \cdot 10^{40}$ \\
$980618 \mathrm{c}$ & 16229.0 & 23 & $7.5 \cdot 10^{-6}$ & $1.0 \cdot 10^{-4}$ & $3.0 \cdot 10^{40}$ & $4.0 \cdot 10^{41}$ \\
$980618 \mathrm{~d}$ & 22593.1 & 20 & $3.0 \cdot 10^{-7}$ & $1.0 \cdot 10^{-5}$ & $1.2 \cdot 10^{39}$ & $4.0 \cdot 10^{40}$ \\
$980618 \mathrm{e}$ & 59884.7 & 18 & $5.5 \cdot 10^{-7}$ & $7.0 \cdot 10^{-6}$ & $2.2 \cdot 10^{39}$ & $2.8 \cdot 10^{40}$ \\
$980622 \mathrm{a}$ & 48596.3 & 24 & $4.5 \cdot 10^{-6}$ & $5.0 \cdot 10^{-5}$ & $1.8 \cdot 10^{40}$ & $2.0 \cdot 10^{41}$ \\
$980622 \mathrm{~b}$ & 68199.1 & 17 & $6.0 \cdot 10^{-7}$ & $3.0 \cdot 10^{-5}$ & $2.4 \cdot 10^{39}$ & $1.2 \cdot 10^{41}$ \\
980625 & 39379.6 & 24 & $4.3 \cdot 10^{-6}$ & $5.0 \cdot 10^{-5}$ & $1.7 \cdot 10^{40}$ & $2.0 \cdot 10^{41}$ \\
980629 & 26706.8 & 17 & $4.7 \cdot 10^{-7}$ & $1.2 \cdot 10^{-5}$ & $1.9 \cdot 10^{39}$ & $4.8 \cdot 10^{40}$ \\
980712 & 78640.1 & 16 & $5.4 \cdot 10^{-7}$ & $2.0 \cdot 10^{-5}$ & $2.2 \cdot 10^{39}$ & $8.0 \cdot 10^{40}$ \\
\hline
\end{tabular}


Fig. 1.- Time histories of 5 bursts from SGR 1627-41. Time resolution $2 \mathrm{~ms}$, photon energy $E_{\gamma}>15 \mathrm{keV}$. Complex temporal structures are evident.

Fig. 2.- The burst on June 18, 16229s UT. Time histories taken in two energy windows G1(15-55 keV) and G2(55-250 keV). A fast strong spectral variability manifests itself in a hardness ratio G1/G2 profile. In time intervals denoted A, B, C, D, E energy spectra were accumulated.

Fig. 3.- The burst on June 18, 16229s UT. Subsequent energy spectra A, B, C, D, E are shifted for clarity by factors of $1 ; 1 / 5 ; 1 / 50 ; 1 / 250 ; 1 / 250$ respectively. The spectra A-D show a variation of parameter $\mathrm{kT}: 22.0 \pm 0.7 ; 18.0 \pm 0.7 ; 28 \pm 1.5 ; 23 \pm 1 \mathrm{keV}$. The last spectrum is fitted by a power law with an index $\alpha=-2.8 \pm 0.3$.

Fig. 4. - The time history of the giant burst on June 18, 6153s UT corrected for dead time. Photon energy $E_{\gamma}>15 \mathrm{keV}$. The rise time is about $100 \mathrm{~ms}$.

Fig. 5.- Energy loss spectra measured in adjacent time intervals A, B, C, D, E and shifted for clarity by factors of $1 / 5 ; 10 ; 1 ; 1 / 25 ; 1 / 125$ respectively. Spectra B, C are strongly affected by pile up effects, especially spectrum B near the peak of the burst. 




Figure 1 


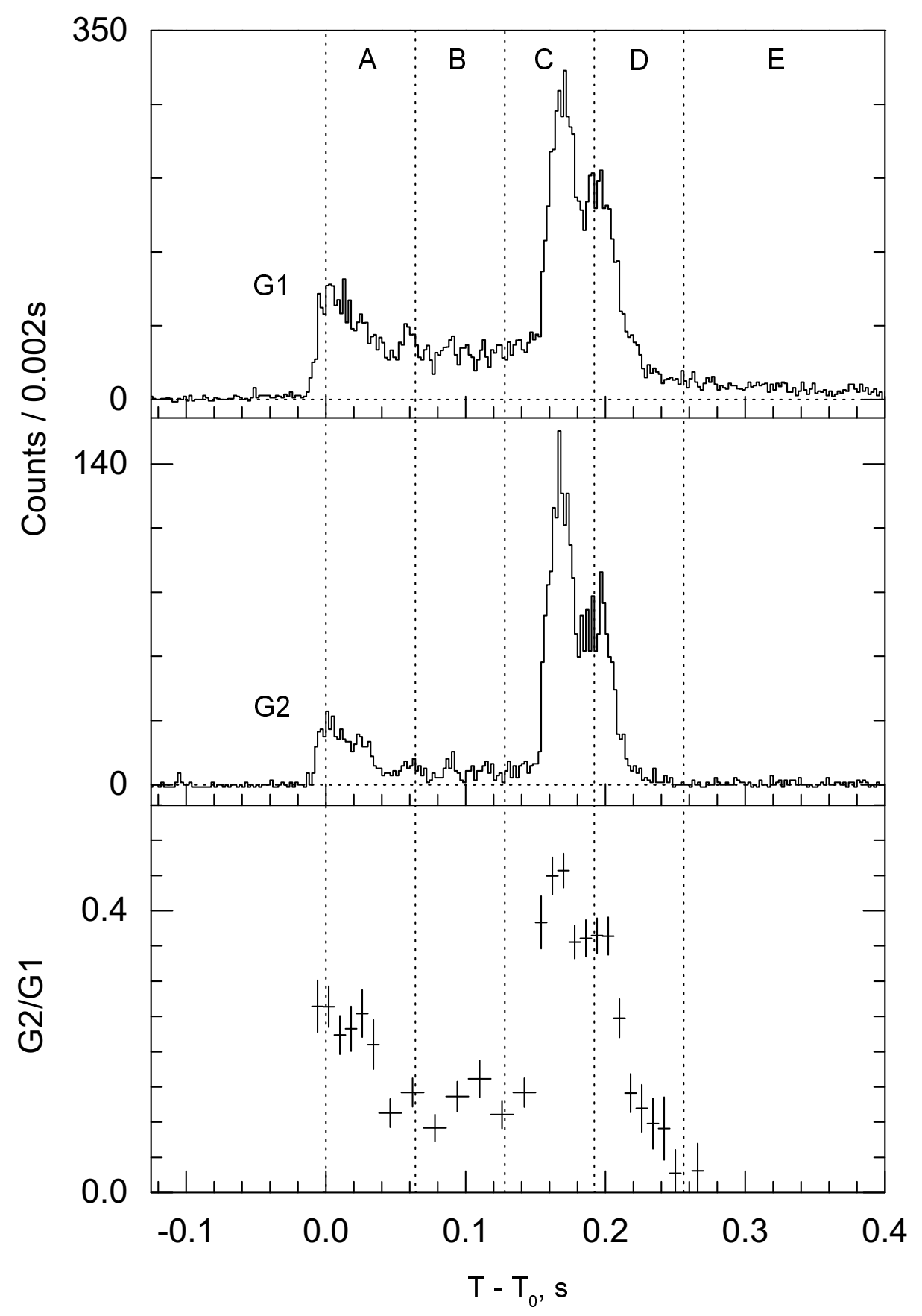

Figure 2 


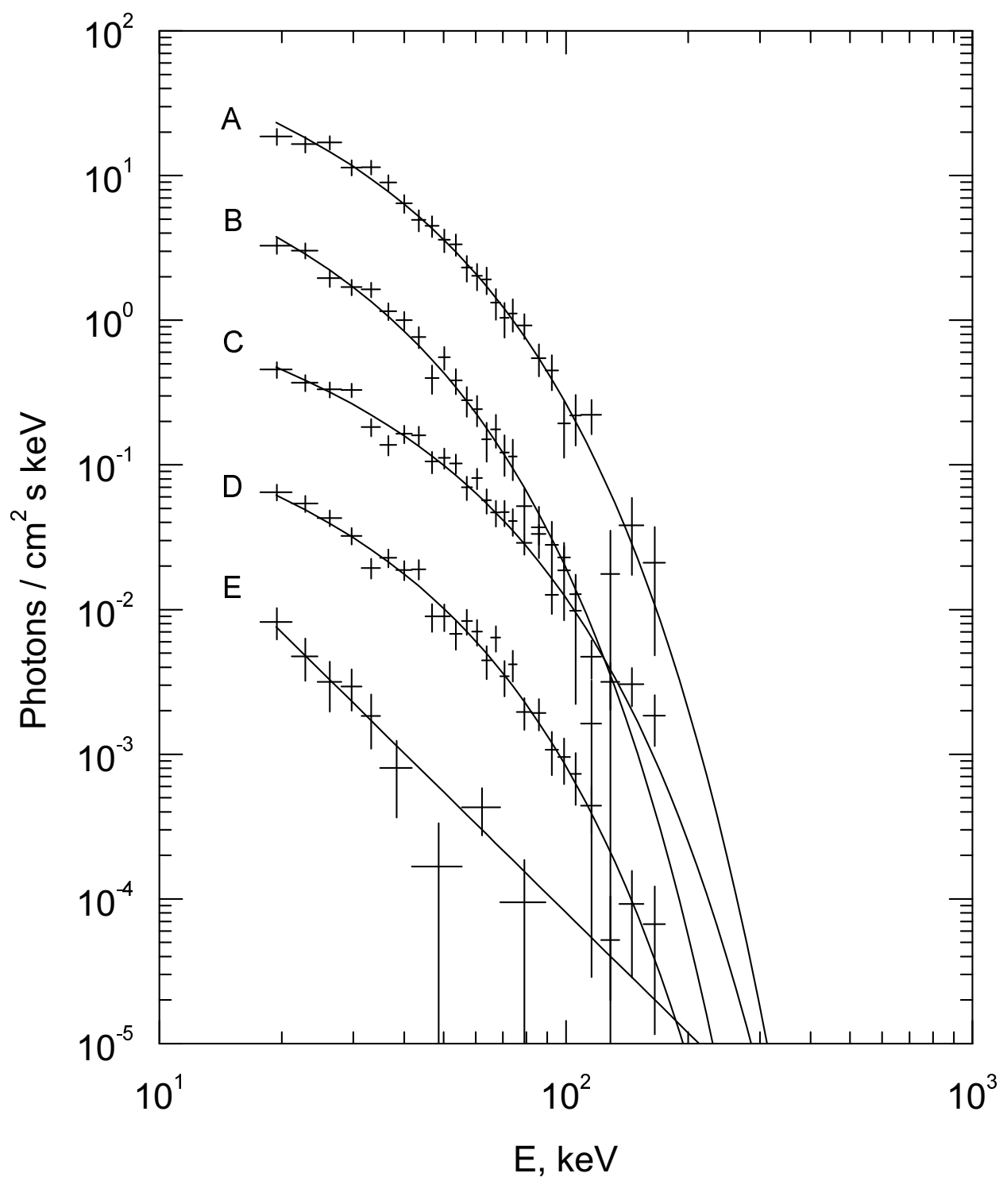

Figure 3 


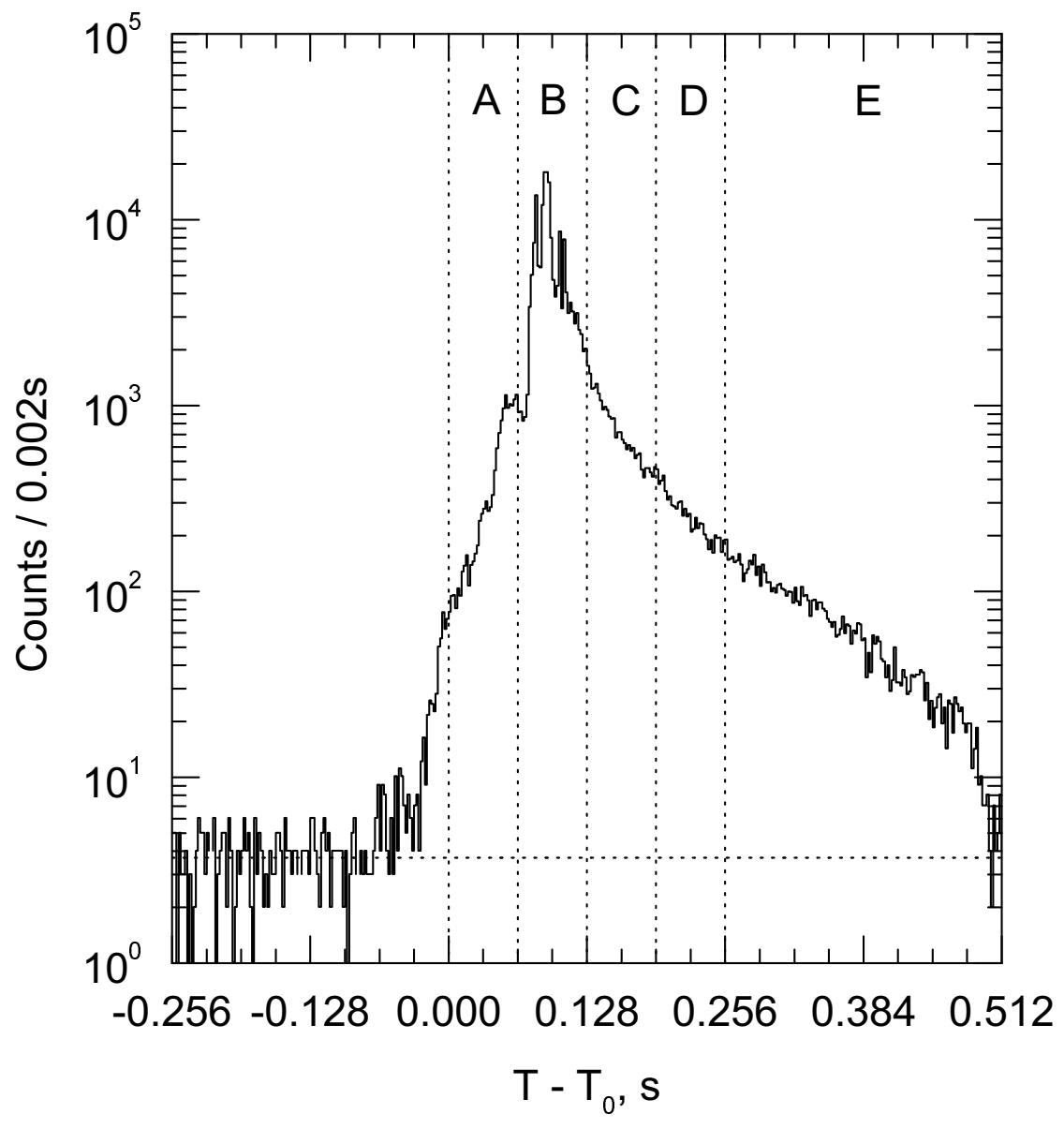

Figure 4 


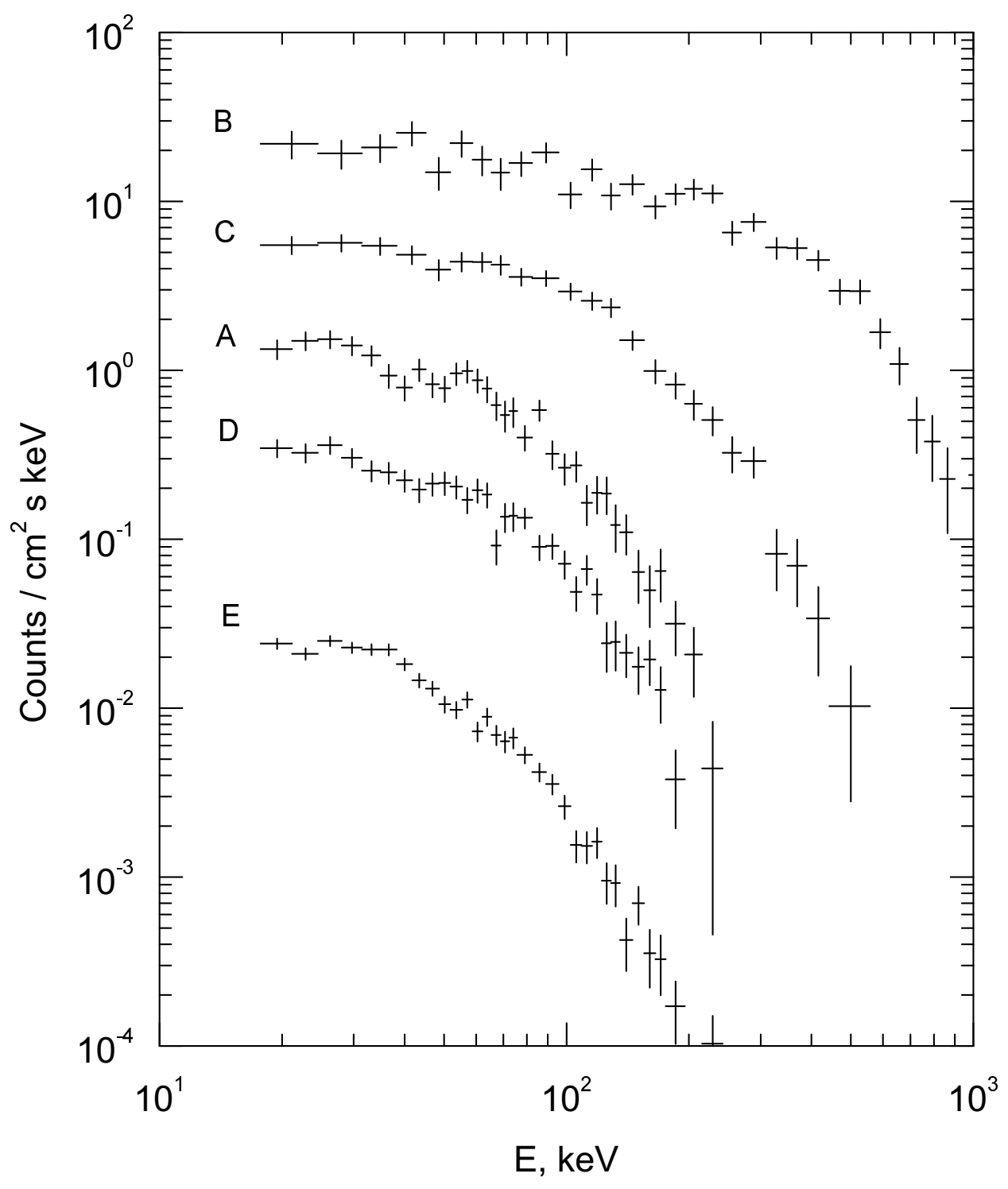

Figure 5 